\title{
MiR-182-5p promotes the Metastasis and Epithelial- mesenchymal Transition in Non-small Cell Lung Cancer by Targeting EPAS1
}

\author{
Wenxiao Yang1\#, Yinan Yin ${ }^{1 \#, ~ L i n g ~ B i ~}{ }^{1}$, Yichao Wang1, Jialin Yao ${ }^{1}$, Ling $\mathrm{Xu}^{1,2}{ }^{\llbracket}$ and Lijing Jiao ${ }^{1,3}$ \\ 1. Department of Oncology, Yueyang Hospital of Integrated Traditional Chinese and Western Medicine, Shanghai University of Traditional Chinese Medicine, \\ Shanghai, China. \\ 2. Cancer Institute of Traditional Chinese Medicine, Shanghai University of Traditional Chinese Medicine, Shanghai, China. \\ 3. Institute of Clinical Immunology, Yueyang Hospital of Integrated Traditional Chinese and Western Medicine, Shanghai University of Traditional Chinese \\ Medicine, Shanghai, China. \\ \#These authors contributed equally to this work.
}

\ Corresponding authors: Lijing Jiao, E-mail: jiaolijing@shyueyanghospital.com; Ling Xu, E-mail: xulq67@aliyun.com.

(c) The author(s). This is an open access article distributed under the terms of the Creative Commons Attribution License (https://creativecommons.org/licenses/by/4.0/). See http://ivyspring.com/terms for full terms and conditions.

Received: 2021.03.14; Accepted: 2021.09.21; Published: 2021.10.17

\begin{abstract}
Background: Dysregulation of microRNAs (miRNAs) is associated with the pathogenesis of non-small cell lung cancer (NSCLC). However, the mechanisms through which miR-182-5p regulate NSCLC progression have not been established. This study aimed at evaluating the expression levels of miR-182-5p in human NSCLC and its function in lung cancer cells. Endothelial PAS Domain-containing protein 1 (EPAS1; also referred to as hypoxia-inducing factor 2A, HIF-2 $\alpha$ ) is a transcription factor that is responsible for induction of genes related to cell survival under hypoxia conditions. Hypoxia, an inherent feature of solid tumors, is associated with aggressive phenotypes, as well as resistance to radiotherapy and chemotherapy, which predict metastasis and poor prognosis.

Methods: The Cancer Genome Atlas (TCGA) dataset was used to investigate the association between miR-182-5p expression and clinicopathological characteristics as well as prognosis of NSCLC patients. Target genes of miR-182-5p were identified using the PITA, miRmap, microT, miRanda, PicTar, and TargetScan prediction tools. Transwell assays were performed to determine the potential functions of miR-182-5p in lung cancer cells. Luciferase reporter assays were performed to analyze regulation of the putative target of miR-182-5p while western blot assays were used to validate the luciferase results.

Results: miR-182-5p was found to be upregulated in NSCLC tissues and acted as an independent prognostic factor for tumor recurrence in NSCLC patients. Functionally, overexpression of miR-182-5p promoted lung cancer cell migration and invasion. Genome-wide gene expression analysis and luciferase report assays revealed that EPAS1 is a direct target of miR-182-5p. EPASI was negatively correlated with miR-182-5p expression in NSCLC tissues. Univariate and multivariate survival analyses identified EPAS1 as an independent prognostic factor for overall survival (OS) in NSCLC.

Conclusions: These findings imply that miR-182-5p promotes NSCLC progression by targeting EPAS1 and is, therefore, a potential indicator of tumor recurrence in NSCLC patients.
\end{abstract}

Key words: EPAS1, miR-182-5p, NSCLC, Metastasis, EMT

\section{Introduction}

Globally, lung cancer is the leading cause of cancer-related mortalities [1]. In 2018, China recorded an estimated 4.3 million new cancer cases and 2.9 million cancer-related mortalities [2]. Over $80 \%$ of lung cancer incidences are NSCLC [3,4]. High frequencies of recurrence and metastasis are highly associated with poor clinical outcomes of lung cancer patients. It has been postulated that miRNA deregulation is correlated with NSCLC progression [5]. MiRNAs inhibit target mRNA based on total or 
partial complementarity by targeting the 3' untranslated region (UTR) of the mRNA [6]. miR-182-5p, a member of the miR-183/96/182 cluster, is related to various cancers [7]. Depending on cancer type, it can function as an oncogene or a tumor suppressor [8]. Suppressed expression levels of miR-182-5p are associated with poor survival outcomes in neuroblastoma [9]. However, miR-182-5p is involved in hepatocellular carcinoma (HCC) metastasis by targeting FOXO3a [10]. While its overexpression promotes NSCLC metastasis by activating the NF-kB signaling pathway [11, 12]. Moreover, upregulated miR-182-5p levels enhance drug resistance in cisplatin-treated A549 by regulating PDCD4 [13].

The association between miR-182-5p expression and NSCLC recurrence has not been elucidated. In this study, the correlation between miR-182-5p and NSCLC recurrence among patients that had been previously subjected to curative surgery was investigated. Underlying mechanisms through which miR-182-5p promote NSCLC progression were also explored.

EPAS1 is a homolog of hypoxia-inducible factor 1a. It plays essential roles in cell survival and invasion through E-cadherin, Vimentin, Ki-67, CD31 [14], and VEGFA [15] [16]. It has been associated with several human cancers [17]. Studies have reported that EPAS1 is the primary target gene of miR-182-5p [18]. However, its underlying mechanisms have not been established [18].

\section{Methods}

\section{Clinical data}

The microRNA-Seq data (513 cases) and the mRNA data (504 cases) and clinical information from The Cancer Genome Atlas (TCGA) database (https:/ / genome-cancer.ucsc.edu; Apache-2.0 licence) were confirmed and downloaded as the study subjects. We enrolled patients with pathologically confirmed stage I-IV NSCLC. The cases were collected from several cancer centers in the United States according to strict standards, and the clinical information of patients was recorded and followed up in detail. Basic clinical information in the database included age, gender, pathological type, TNM stage (Table S1 \& 2). GSE36681 (Normal 103 and Tumor 103) was downloaded from the Gene Expression Omnibus database (GEO, https://www.ncbi.nlm.nih.gov/ geo/). GEO2R was used to determine the gene expression profiles of lung adenocarcinoma (LUAD) and normal control (NC) samples and identify genes that were differentially expressed. Target genes of miR-182-5p were identified using the PITA [19],
miRmap [20], DIANA-microT [21], miRanda [22], PicTar [23], and TargetScan [24] prediction tools. Analysis of differentially expressed genes in NSCLC was performed using Gene Expression Profiling Interactive Analysis 2 (GEPIA2) [25]. Correlation analysis between miR-182-5p and target genes was performed using ENCORI [26]. Disease-free survival (DFS) was defined as the time between surgery and the first event of either disease recurrence or death from any cause. OS was defined as the time between the date of diagnosis and death from any cause.

\section{Cell culture}

NSCLC cell lines (NCI-H1975, NCI-H460, A549, 95-D), human bronchial epithelial cells (16HBE) and human lung epithelial cell lines (BEAS-2B) were purchased from the Chinese Academy of Sciences Cell Bank. Cells were cultured in RPMI 1640 medium (Corning, USA) supplemented with 10\% Fetal Bovine Serum (FBS) (Gibco, USA) and 100 units per ml of penicillin-streptomycin solution. The 293T cells were cultured in Dulbecco's Modified Eagle Medium supplemented with 10\% FBS (Gibco, USA). Incubation was performed in a humidified atmosphere containing $5 \% \mathrm{CO}_{2}$ at $37{ }^{\circ} \mathrm{C}$. Mycoplasma test of all cells was negative.

\section{miRNA and mRNA quantitative real-time PCR}

Total RNA was extracted from NSCLC cells using the TRIzol reagent [27]. Briefly, $2 \mu \mathrm{g}$ of extracted RNA were reverse transcribed into complementary DNA (cDNA) with the application of reverse transcriptase (Thermo Fisher, USA). The qRT-PCR reactions were performed using Power SYBR Green PCR Master Mix (Kapa biosystems, USA), and GAPDH was used as the internal control. Then, miRNAs were isolated from total RNA using a High Pure miRNA isolation kit (TIANGEN, KR211-01, China) followed by their transcription through RT-PCR using a TaqMan MicroRNA Reverse Transcription kit (TIANGEN Technologies, FP411-01, China). Data were analyzed using the comparative $\mathrm{Ct}$ method $(2-\Delta \Delta C t)$. Three experiments were performed for each clone. Primer sequences used in this study are presented in the following information (Table 1 \& 2).

\section{Invasion and migration assay}

Transfected cells $\left(2 \times 10^{4}\right)$ in $200 \mu$ l serum free medium were added to the upper compartment of chambers (Corning, NY, USA) which were pre-coated with Matrigel (Corning, NY, USA). Chambers were placed in 24-well plates with $0.5 \mathrm{ml}$ of $15 \%$ FBS medium. After $24 \mathrm{~h}$ of incubation at $37^{\circ} \mathrm{C}$, chambers were obtained, the medium was discarded after which they were washed using PBS (5 times, each for 
$5 \mathrm{~min})$. Immediately, chambers were fixed in 24-well plates containing $300 \mu \mathrm{l}$ of $4 \%$ PFA for $30 \mathrm{~min}$. Then, they were washed 3 times using PBS and stained with $300 \mu \mathrm{l}$ crystal violet $(0.1 \%)$ for another $90 \mathrm{~min}$. Cells in the wells were washed using the PBS buffer. Then, cells in the upper compartment were wiped away while cells in the lower compartment were observed by light microscopy. The number of five high power fields per chamber were counted and mean value calculated. The migration assay was similarly performed as the invasion assay, except that no Matrigel was used.

Table 1. Primer sequences for mRNA expression

\begin{tabular}{|c|c|c|}
\hline Gene name & & Sequence \\
\hline \multirow[t]{2}{*}{ E-cadherin } & forward & CCTTGTGATCCGCCTGCCTTG \\
\hline & reverse & СТGCCTGCCTGCСТTCTGATTAC \\
\hline \multirow{2}{*}{ N-cadherin } & forward & CAGAATCGTGTCTCAGGCTCCAAG \\
\hline & reverse & CTGCGTTCCAGGCTGGTGTATG \\
\hline \multirow[t]{2}{*}{ Twist } & forward & CGACGACAGCCTGAGCAACAG \\
\hline & reverse & TCCTCGTAAGACTGCGGACTCC \\
\hline \multirow[t]{2}{*}{ Snail } & forward & TTACCTTCCAGCAGCCCTAC \\
\hline & reverse & CTTTCGAGCCTGGAGATCCT \\
\hline \multirow[t]{2}{*}{ MMP-2 } & forward & TTCCAAGTCTGGAGCGATGT \\
\hline & reverse & CAGAAGCCGTACTTGCCATC \\
\hline \multirow[t]{2}{*}{ MMP-9 } & forward & GCCACTACTGTGCCTTTGAG \\
\hline & reverse & TCAAAGACCGAGTCCAGCTT \\
\hline \multirow[t]{2}{*}{ Vimentin } & forward & CGCCAACTACATCGACAAGG \\
\hline & reverse & TGAAGCATCTCCTCCTGCAA \\
\hline \multirow[t]{2}{*}{ GAPDH } & forward & CAGACCACAGTCCATGCCATCAC \\
\hline & reverse & GACGCCTGCTTCACCАССТTC \\
\hline
\end{tabular}

Table 2. Sequences of the miR-182-5p mimic, inhibitor, and NC

\begin{tabular}{ll}
\hline Name & Sequences $\left(5^{\prime}\right.$ to $\left.3^{\prime}\right)$ \\
\hline miR-182-5p NC & UUCUCCGAACGUGUCACGUTT \\
miR-182-5p mimic & UUUGGCAAUGGUAGAACUCACACU \\
miR-182-5p NC & CAGUACUUUUGUGUAGUACAA \\
miR-182-5p inhibitor & AGUGUGAGUUCUACCAUUGCCAAA \\
\hline
\end{tabular}

\section{Western blot analysis}

Cells were lysed using the RIPA buffer (Sangon, China) containing a Proteinase inhibitor (Roche, Switzerland) and a Pierce phosphatase inhibitor (Thermo Fisher, USA) [27]. Protein extracts were boiled in a loading buffer followed by separation on a $15 \%$ sodium dodecyl sulfate-polyacrylamide gel electrophoresis platform (SDS-PAGE). Separated protein bands were transferred onto polyvinylidene fluoride membranes. Primary antibodies against EPAS1 (7096s, CST, USA), GAPDH (2118S, CST, USA), N-cadherin (13116S, CST, USA), Vimentin (5741S, CST, USA), E-cadherin (3195S, CST, USA), MMP-9 (13667S, CST, USA), MMP-2 (40994S, CST, USA), Snail (3879S, CST, USA), and Twist (69366S, CST, USA) were diluted at a ratio of 1:1000 in accordance with the manufacturer's instructions. Then, membranes were probed with a goat anti-rabbit
IgG highly cross-adsorbed secondary antibody (HSA0003, maibio, China) for $2 \mathrm{~h}$ at room temperature after which they were exposed to the enhanced chemiluminescence (ECL) reagent for visualization.

\section{Luciferase reporter assay}

The 293T cells were cultured in 24-well plates and randomly allocated into four groups for transfection studies using the Lip3000 transfection kit (Thermo Fisher Scientific, USA). Plasmid sequences used in this study are shown in the following information (Table 3). For each group, co-transfection was performed using a control plasmid (40 ng), a recombinant plasmid (800 $\mathrm{ng}$ ), and a final mimics concentration of $20 \mathrm{nmol} / \mathrm{L}$. Each group was set with 5 multiple pores. Cells were lysed, after which positive transfection was detected using the double luciferase assay kit (Biology of Hanheng, China) after $48 \mathrm{~h}$ of transfection. After measuring using a chemiluminescence instrument, the blank hole's basal signal was subtracted from the data of each hole. The firefly fluorescence signal generated by the plasmid was used as the control. Relative fluorescence activities were divided by sea kidney's fluorescence signal and normalized for each group.

Table 3. Sequence of plasmids

\begin{tabular}{ll}
\hline Name & Sequence (5 'to 3 ') \\
\hline miR-182-5p NC & UUCUCCGAACGUGUCACGUTT \\
miR-182-5p mimics & ACGUGACACGUUCGGAGAATT \\
& UUUGGCAAUGGUAGAACUCACACU \\
EPAS1-WT-hsa-miR-182-5p & UGUGAGUUCUACCAUUGCCAAAUU \\
& UUGAGACUUAUUGCCAA \\
EPAS1-MUT-hsa-miR-182-5p & GCAAAGTGUUTAACGGTT \\
& UUGGCAAUG-GUAGAACU \\
\hline
\end{tabular}

\section{Data analysis}

Univariate Cox regression analysis was performed to identify primary prognostic factors while multivariate Cox regression analysis and construction of the K-M survival curve were performed to establish the risk score model, and identify independent prognostic factors. Receiver operating characteristic curve (ROC curve) analysis was performed to estimate the prognostic power of the risk score model. Results of the receiver operating characteristic curve (ROC) in IBM SPSS Statistics version 24.0 (SPSS Inc., Chicago, IL, USA) were used to determine the diagnostic critical value and obtain the AUC (Area under the curve). The Youden Index was calculated as: Youden index $=$ sensitivity + specificity -1 . The maximum value of the Youden index is the optimal miR-182-5p and EPAS1 bound value. Cut-off values under the ROC curve were grouped to determine the relationship between their 
expression levels and lung cancer prognosis. The GraphPad Prism version 7.0 (GraphPad Software, USA) was used to analyze data regarding the function of miR-182-5p, which were presented as means \pm standard deviation (SD). Differences between groups were determined using the GraphPad Prism software. $p \leq 0.05$ was set as the threshold for statistical significance.

\section{Results}

\section{miR-1 82-5p expression was associated with tumor recurrence in NSCLC}

The TCGA database was used to establish the relationship between miR-182-5 $p$ expression levels and tumor metastasis, recurrence, and long-term survival in lung adenocarcinoma patients. Among the 46 matched lung adenocarcinoma samples, expression levels of miR-182-5p in normal lung tissues were significantly elevated when compared to lung adenocarcinoma tissues $(\mathrm{p}<0.0001)$ (Figure 1A). Expression levels of miR-182-5p in lung tissues of 513 patients with unpaired lung adenocarcinoma (25183 \pm 752.90) were significantly higher than those of 46 patients with normal lung tissues $(p<0.0001)$ (Figure 1B). In addition, we downloaded the GSE36681 data from the GEO database for validation $(p<0.0001$, Figure S1). At the same time, in 45 matched samples of lung squamous cell carcinoma (LUSC) patients from the TCGA database, expression levels of miR-182-5p in normal lung tissues were significantly higher than those in lung squamous cell carcinoma tissues $(p<0.0001$; Figure S2). Expression levels of miR-182-5p in lung tissues of 478 unmatched patients with lung squamous cell carcinoma were significantly higher than those of 45 normal lung tissues $(p<0.0001$; Figure S3). Sensitivity and specificity of miR-182-5p were $30.7 \%$ and $78.52 \%$, respectively, with a corresponding cut-off value of 32439.93 (Figure 1C \& D).

Out of the 513 patients with lung adenocarcinoma, 396 patients $(77.19 \%)$ were in the miR-182-5p low expression group (Figure 1D) and 117 patients $(22.80 \%)$ were in the miR-182-5p high expression group. The DFS was 1447 days in the miR-182-5p low expression group versus 921 days. In the miR-182-5p high expression group $(\mathrm{HR}=0.7125$, (95\% CI: 0.4997-1.016; $\mathrm{p}=0.0394$ ) (Figure 1E). In the Cox univariate and multivariate regression analyses, $\mathrm{T}$ stage was established as a prognostic factor. The risk of recurrence and metastasis for $\mathrm{T} 3+\mathrm{T} 4$ stages was 1.881 times higher than that for $\mathrm{T} 1+\mathrm{T} 2$ stages (95\% CI: 1.254-2.823; $r=0.0 .632 ; p<0.05$ ). Meanwhile, the risk of recurrence and metastasis in lung adenocarcinoma patients with low expression levels of miR-182-5p was 0.691 times higher than that of patients with high expression levels $(95 \%$ CI: 1.058-2.049). This finding suggests that miR-182-5p expression is an independent prognostic factor $(\mathrm{r}=$ 0.369; $\mathrm{p}<0.05$; Table 4).
A

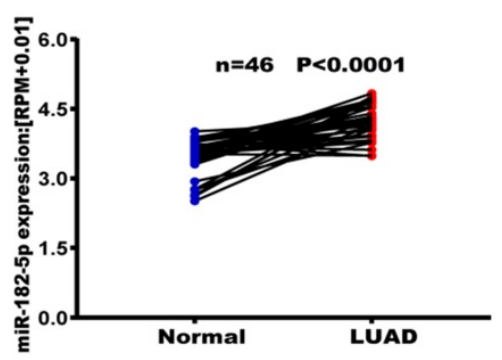

D

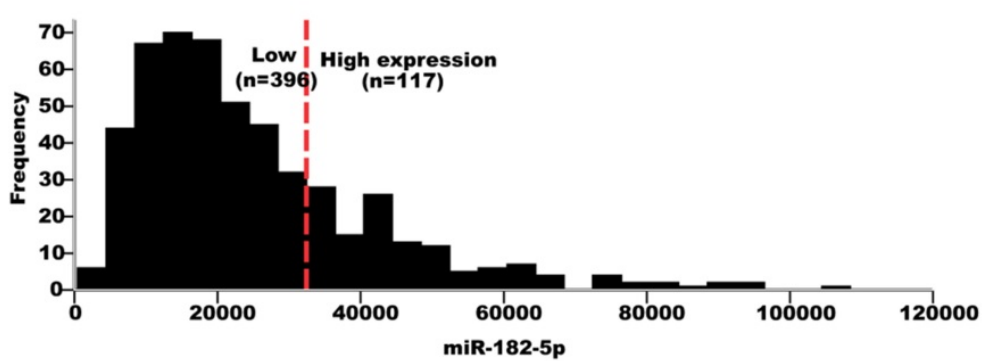

B

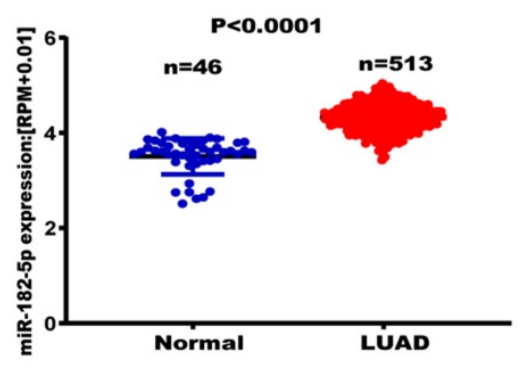

E

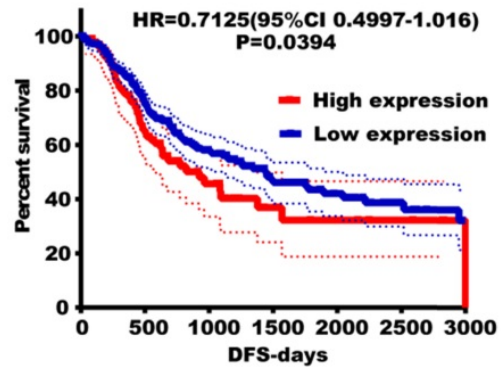

Figure 1. TCGA analysis of expression levels of miR-182-5p in LUAD tissues. (A) TCGA analysis of the expression levels of miR-182-5p in paired LUAD tissues. (B) TCGA analysis of the expression levels of miR-182-5p in unpaired LUAD tissues. (C) ROC curve used to obtain a cut-off value of miR-182-5p in LUAD. (D) LUAD patients categorized based on the cut-off value of miR-182-5p. (E) Kaplan-Meier analysis of the association between miR-182-5p expression and DFS. 


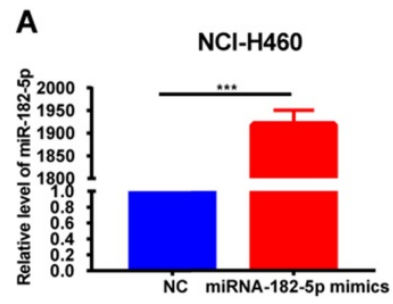

B

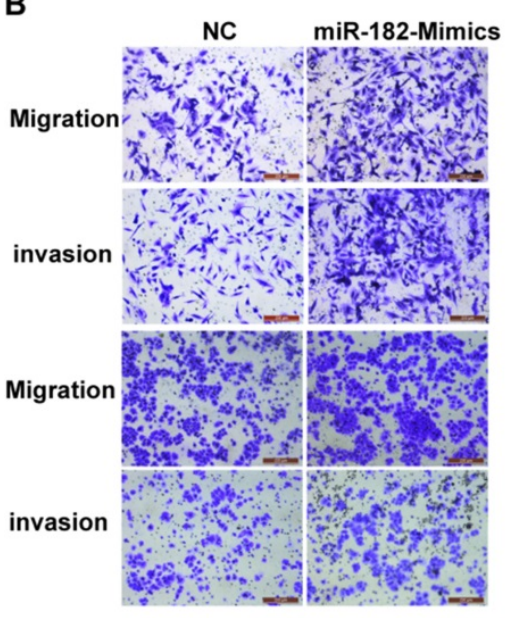

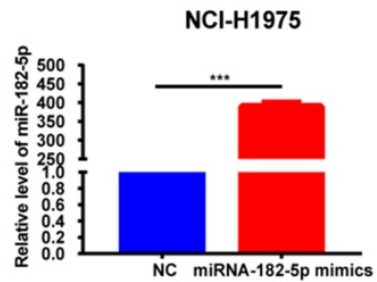
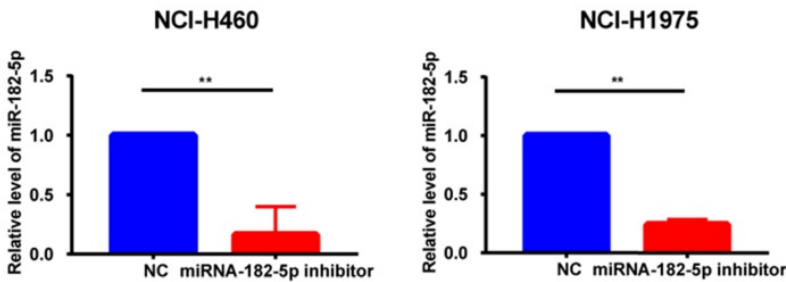

C
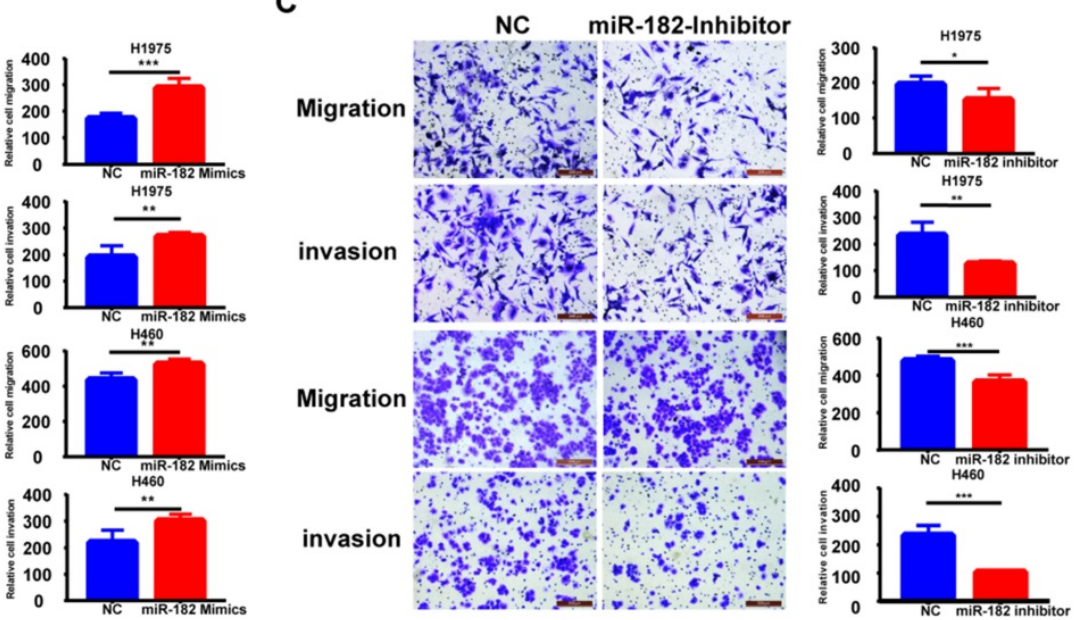

Figure 2. Effect of altered miR-182-5p expression levels on NSCLC metastasis. (A) Expression levels of miR-182-5p in different cell lines after transfection with miR-182-5p mimics/inhibitor. (B) Effect of miR-182-5p over-expression on lung cancer cell invasion and migration. (C) Effect of miR-182-5p under-expression on lung cancer cell invasion and migration. ( $\left.{ }^{*} \mathrm{p}<0.05, * * \mathrm{p}<0.01, * * * \mathrm{p}<0.001\right)$.

Table 4. Cox regression analysis of miR-182-5p expression as a tumor recurrence predictor

\begin{tabular}{|c|c|c|c|c|}
\hline \multirow[t]{2}{*}{ Variables } & \multicolumn{2}{|c|}{$\begin{array}{l}\text { Univariate Cox's regression } \\
\text { analysis }\end{array}$} & \multicolumn{2}{|l|}{$\begin{array}{l}\text { Multivariate Cox's } \\
\text { regression analysis }\end{array}$} \\
\hline & RR $(95 \% \mathrm{CI})$ & p-value & $\mathrm{RR}(95 \% \mathrm{CI})$ & p-value \\
\hline \multicolumn{5}{|l|}{ Age (years) } \\
\hline$<65$ versus $>65$ & $1.203(0.891-1.6223)$ & 0.227 & NA & NA \\
\hline \multicolumn{5}{|l|}{ Gender } \\
\hline Male versus Female & $0.953(0.713-1.275)$ & 0.746 & NA & NA \\
\hline \multicolumn{5}{|l|}{ Pathological stage } \\
\hline $\begin{array}{l}\text { I/IIIa versus IIIb+IV } \\
\text { T stage }\end{array}$ & $1.385(0.771-2.488)$ & 0.276 & NA & NA \\
\hline $\mathrm{T} 1+\mathrm{T} 2$ versus $\mathrm{T} 3+\mathrm{T} 4$ & $1.956(1.305-2.931)$ & 0.001 & $1.881(1.254-2.823)$ & 0.002 \\
\hline \multicolumn{5}{|l|}{ N stage } \\
\hline $\begin{array}{l}\text { Negative versus } \\
\text { Positive }\end{array}$ & 1.484 (1.103-1.995) & 0.009 & NA & NA \\
\hline \multicolumn{5}{|l|}{ M stage } \\
\hline $\begin{array}{l}\text { Negative versus } \\
\text { Positive } \\
\text { miR-182-5p expressi }\end{array}$ & $1.114(0.812-1.528)$ & 0.503 & NA & NA \\
\hline High versus Low & $0.711(0.514-0.985)$ & 0.040 & $0.691(0.498-0.960)$ & 0.028 \\
\hline
\end{tabular}

RR: risk ratio; CI: confidence interval; NA: not analyzed.

\section{miR-1 82-5p promotes NSCLC migration and invasion}

The expression of miR-182-5p was negatively correlated with tumor recurrence and metastasis. Therefore, we hypothesized that miR-182-5p inhibits NSCLC cell migration and invasion. Expression levels of miR-182-5p in commonly used human NSCLC cell lines and human lung epithelial cells were determined to test this hypothesis. Expression levels of miR-182-5p in 95-D, NCI-H1975, A549, and NCI-H460 cells were significantly elevated when compared to normal lung cells (16HBE and BEAS-2B) ( $p<0.05$; Figure S4). Transfection efficiencies of miR-182-5p mimics/inhibitor in NCI-H1975 and NCI-H460 cell lines were confirmed by qRT-PCR (Figure 2A). NSCLC cell invasion and migration were promoted by elevated expression levels of miR-182-5p and inhibited by its suppressed expression (Figure 2B \& C).

\section{Identification of EPASI as a direct target of miR-182-5p in NSCLC cells}

Six different miRNA target prediction databases were used to select 121 common target genes to establish the molecular mechanisms involved in regulation of lung cancer cell migration and invasion by miR-182-5p (Table S3). Differentially expressed genes in lung adenocarcinoma were obtained from the TCGA project through GEPIA2 database analysis [25]. There were 26 differentially expressed genes that were found to be regulated by miR-182-5p. Expression levels of EPAS1 in the gene list were significantly lower than those of adjacent tissues (Figure 3A). EPAS1 was negatively correlated with miR-182-5p expression in NSCLC tissues $(r=-0.146, p$ $=0.000934$; Figure 3B). Then, the Luciferase reporter assay revealed that wild type (WT) or mutant (Mut) 3'UTR of EPAS1 were successfully co-transfected with miR-182-5p mimics into the 293T cell line (Figure 3C). 
These findings imply that, compared to the miR-NC group, luciferase activity of the WT 3'UTR of EPAS1 was enhanced by increasing the expression of miR-182-5p. However, that of Mut 3'UTR of EPAS1 was unaffected by miR-182-5p's overexpression (Figure 3D). These findings confirm that EPAS1 is the target gene of miR-182-5p.

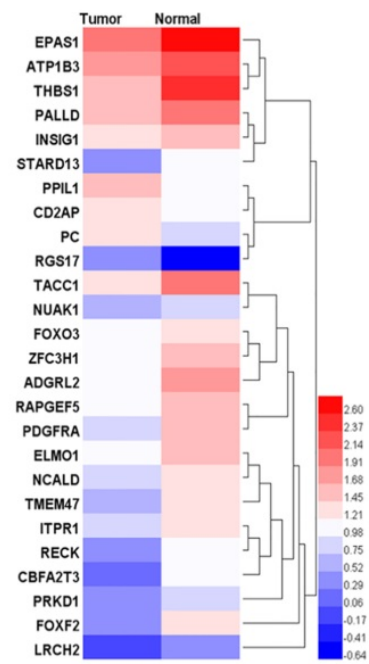

C

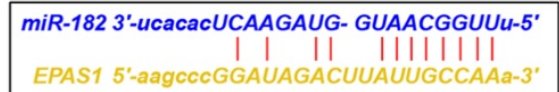

\section{EPAS1 expression is associated with poor survival in NSCLC}

EPAS1 expression levels were found to be suppressed in paired and unpaired NSCLC tissues (n = 513) compared to the 46 adjacent normal tissues (Figure 4A \& B). An EPAS1 cut-off value was obtained

B

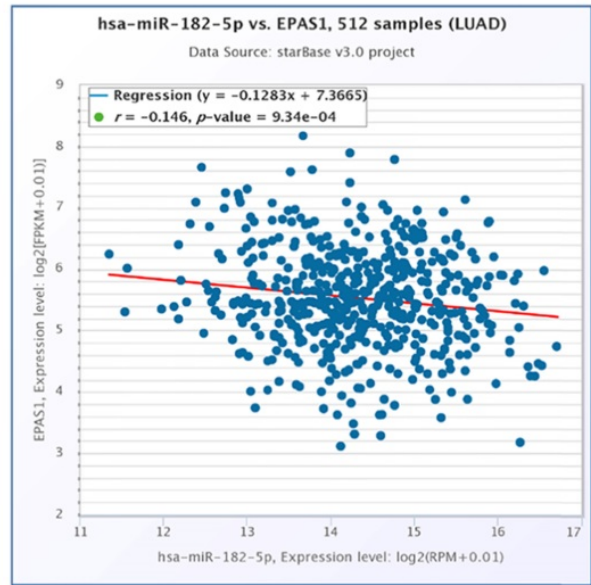

D

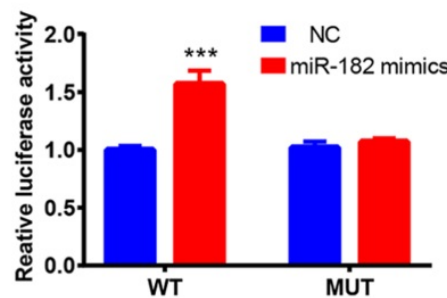

Figure 3. Identification of EPASI as a direct target of miR-182-5p in NSCLC cells. (A) Heatmap of 26 differentially expressed genes regulated by miR-182-5p in LUAD. (B) Pearson correlation analysis of the correlation between miR-182-5p and EPAS1 expressions in LUAD tissues. (C) Binding sites of miR-182-5p to WT or Mut 3' UTR of EPAS1. (D) Luciferase activities of WT or Mut 3' UTR of EPAS1 after co-transfection with miR-182-5p mimics and WT or Mut 3'UTR of EPAS1 in NSCLC cells. (**** $<0.001$ ).
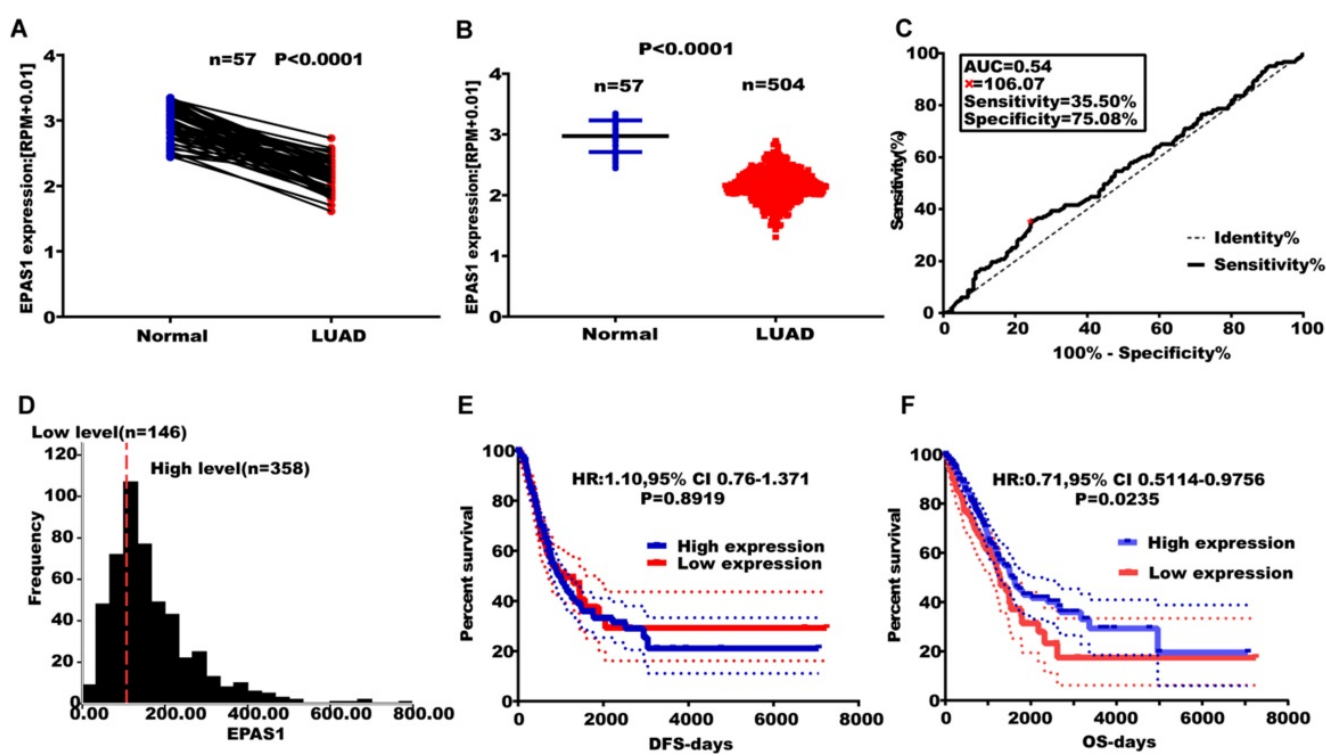

Figure 4. Effect of altered EPASI expression levels on LUAD metastasis. (A) TCGA analysis of the expression levels of EPAS1 in paired LUAD tissues. (B) TCGA analysis of the expression levels of EPAS1 in unpaired LUAD tissues. (C) ROC curve used to obtain a cut-off value of EPAS1 in LUAD. (D) LUAD patients, categorized based on the cut-off value of EPASI. (E) Kaplan-Meier analysis of the association between EPAS1 expression and DFS as well as tumor recurrence. (F) Kaplan-Meier analysis of the association between EPASI expression and OS as well as poor survival. 
using LUAD, based on OS time, survival status, and EPAS1 expression levels. Sensitivity and specificity of EPAS1 were $35.50 \%$ and $75.08 \%$, respectively, with a corresponding cut-off value of 106.07 (Figure 4C \& D). Based on the cut-off value, patients were grouped into the high and low-expression groups. Out of the 504 patients with lung adenocarcinoma, 358 patients were in the miR-182-5p low expression group, 146 patients were in the miR-182-5p high expression group (Figure $4 \mathrm{D})$. The OS of the low expression group was not associated with age, gender, pathological stage, $\mathrm{T}$ stage, $\mathrm{N}$ stage, and $\mathrm{M}$ stage of patients ( $\mathrm{p}>0.05)$. Log-rank test revealed that patients with elevated EPAS1 expression levels resulted in a better overall survival outcome $(p=0.0235)$. However, there were no significant differences in DFS for patients in both expression groups $(\mathrm{p}=0.8919)$ (Figure $4 \mathrm{E} \& \mathrm{~F})$. Cox univariate regression analysis revealed that clinical stage, $\mathrm{T}$ stage, $\mathrm{N}$ stage, and EPAS1 expression levels were the primary factors affecting lung adenocarcinoma recurrence and metastasis. The subsequent multivariate regression analysis revealed that $\mathrm{T}$ stage, $\mathrm{N}$ stage, and EPAS1 expression were independent prognostic factors for lung adenocarcinoma $(\mathrm{p}<0.05)$. The death risk for lung cancer patients with suppressed EPAS1 expression levels was found to be 1.478 times higher than that of patients with elevated EPAS1 expression levels (95\% CI: 1.087-2.008; Table 5).

\section{miR-182-5p promotes epithelial-mesenchymal transition in NSCLC by targeting EPAS 1}

Both qRT-PCR and Western blot analyses revealed that overexpression of miR-182-5p suppressed the expression levels of EPAS1 in NCIH1975 and NCI-H460 cells compared to the miR-NC group (Figure 5A \& C). The EMT process was closely correlated with cancer cell migration and invasion.
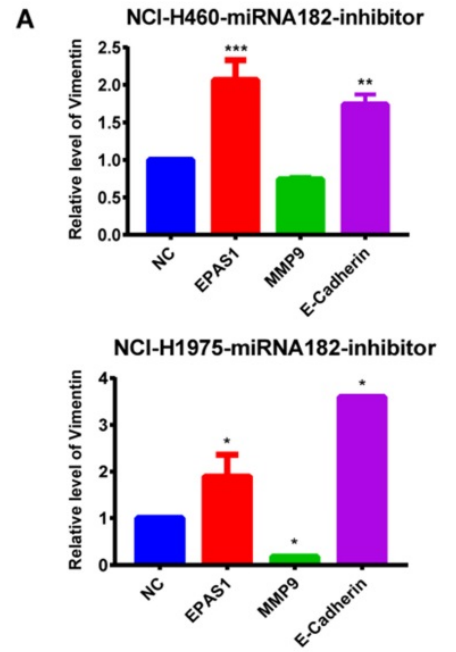

B
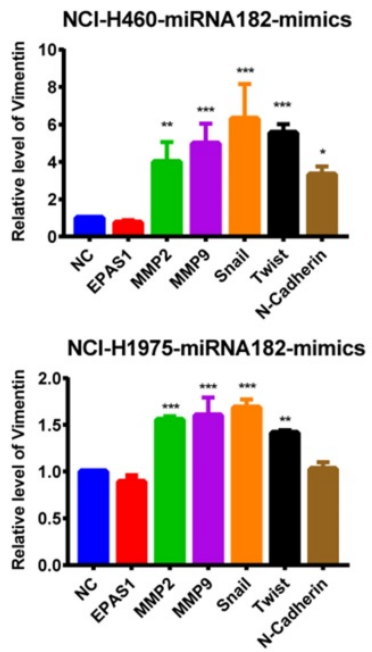

C

\section{GAPDH}

EPAS1

D
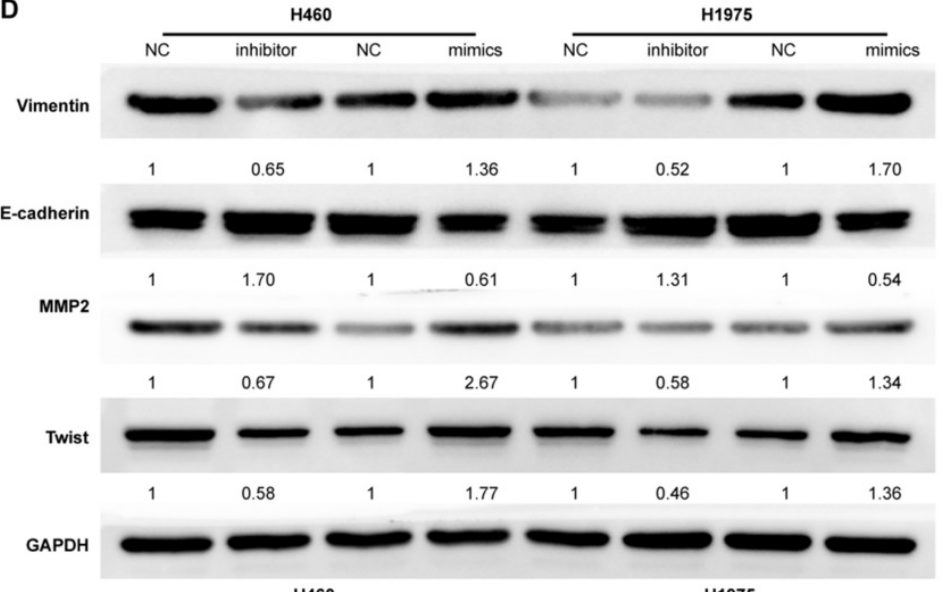

GAPDH
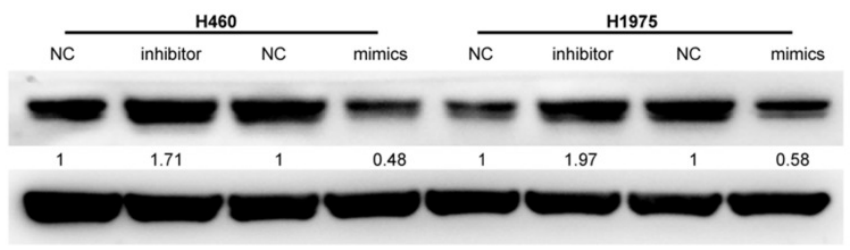

N-cadhe
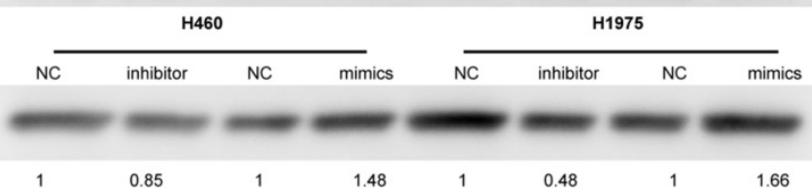

MMP9

\begin{tabular}{l} 
MMP9 \\
Snail \\
\hline POPH
\end{tabular}

GAPDH

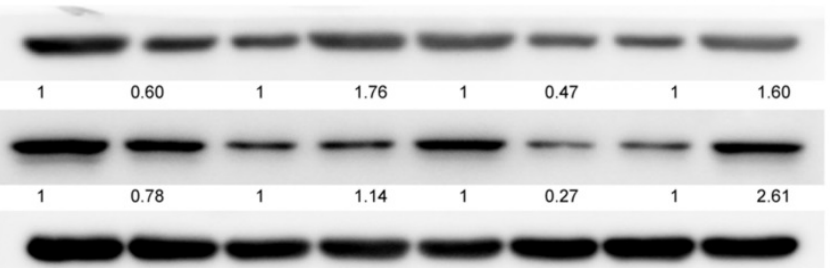

Figure 5. EMT process and EPASI expression analyses by qRT-PCR and western blot. (A) Effect of down-regulated miR-182-5p on the EMT process and EPAS1 expression in $\mathrm{NCl}-\mathrm{H} 460$ and $\mathrm{NCl}-\mathrm{H} 1975$ cells as determined by qRT-PCR. (B) Effect of overexpressed miR-182-5p on the EMT process and EPAS1 expression in NCI-H460 and $\mathrm{NCl}-\mathrm{H} 1975$ cells as determined by qRT-PCR. (C) Effect of down-regulated and overexpressed miR-182-5p on EPAS1 expression in NCl-H460 and NCl-H1975 cells as determined by western blot. (D) Effect of down-regulated and overexpressed miR-182-5p on EMT expression in $\mathrm{NCl}-\mathrm{H} 460$ and $\mathrm{NCl}-\mathrm{H} 1975$ cells as determined by western blot. $\left({ }^{*} \mathrm{p}<0.05,{ }^{*} \mathrm{p}<0.01,{ }^{* * *} \mathrm{p}<0.001\right)$. 
Quantification of mRNA expression levels of EMTassociated genes by qRT-PCR revealed that the epithelial marker, E-cadherin, was down-regulated. In contrast, the mesenchymal marker, MMP-9, was up-regulated in NCI-H460 and NCI-H1975 cells treated with a miR-182-5p inhibitor (Figure 5A). MMP-2, MMP-9, Snail, Twist, and N-cadherin were significantly up-regulated in NCI-H460 and NCIH1975 cells treated with miR-182-5p mimics (Figure 5B). Inhibition of miR-182-5p suppressed protein expression levels of Vimentin, N-cadherin, MMP-2, MMP-9, Snail, and Twist but increased the expression levels of E-cadherin compared to the NC group in NCI-H460 and NCI-H1975 cells (Figure 5D). However, these changes were reversed by overexpression of miR-182-5p. These findings show that cells underwent the EMT process after transfection with miR-182-5p mimics/inhibitor.

Table 5. Cox regression analysis of prognostic factors for overall survival of lung adenocarcinoma

\begin{tabular}{|c|c|c|c|c|}
\hline \multirow[t]{2}{*}{ Variables } & \multicolumn{2}{|c|}{$\begin{array}{l}\text { Univariate Cox's regression } \\
\text { analysis }\end{array}$} & \multicolumn{2}{|l|}{$\begin{array}{l}\text { Multivariate Cox's } \\
\text { regression analysis }\end{array}$} \\
\hline & RR (95\%CI) & p-value & RR $(95 \% \mathrm{CI})$ & p-value \\
\hline \multicolumn{5}{|l|}{ Age (years) } \\
\hline$<65$ versus $>65$ & $0.853(0.633-1.148)$ & 0.293 & NA & NA \\
\hline \multicolumn{5}{|l|}{ Gender } \\
\hline Male versus Female & $0.942(0.704-1.260)$ & 0.688 & NA & NA \\
\hline \multicolumn{5}{|l|}{ Pathological stage } \\
\hline $\begin{array}{l}\text { I/ IIIa versus IIIb+IV } \\
\text { T stage }\end{array}$ & $2.171(1.401-3.364)$ & 0.001 & NA & NA \\
\hline $\mathrm{T} 1+\mathrm{T} 2$ versus $\mathrm{T} 3+\mathrm{T} 4$ & $2.411(1.666-3.487)$ & 0.000 & $2.170(1.493-3.154)$ & $<0.001$ \\
\hline \multicolumn{5}{|l|}{ N stage } \\
\hline $\begin{array}{l}\text { Negative versus } \\
\text { Positive }\end{array}$ & $2.550(1.903-3.417)$ & 0.000 & $2.298(1.709-3.091)$ & $<0.001$ \\
\hline \multicolumn{5}{|l|}{ M stage } \\
\hline $\begin{array}{l}\text { Negative versus } \\
\text { Positive }\end{array}$ & $0.952(0.847-1.070)$ & 0.409 & NA & NA \\
\hline \multicolumn{5}{|l|}{ EPAS1 expression } \\
\hline High versus Low & 1.407 (1.038-1.906) & 0.028 & $1.478(1.087-2.008)$ & 0.013 \\
\hline
\end{tabular}

\section{Discussion}

Abnormal miRNA expression levels in early stages of lung cancer have been correlated with prognosis [28]. Besides, miRNAs have a high pooled sensitivity and specificity in lung cancer diagnosis and treatment [29].

miR-182-5p is a small non-coding RNA that regulates gene expression by directly binding the 3'-UTR of the target mRNA. It regulates multiple biological processes by regulating over 1000 target genes involved in cell cycle, proliferation, apoptosis, metastasis, and metabolism [30-32]. Moreover, it has been shown to promote the occurrence and development of breast cancer [33], lung cancer [34], thyroid cancer [35], medulloblastoma [36], and glioma [37], thereby leading to poor prognosis. It is usually highly expressed in lung cancer and tuberculosis patients [38]. Some studies postulate that serum miR-182-5p is highly sensitive and specific as a potential biomarker for early lung cancer detection [28]. Moreover, miR-182-5p has been shown to play a dual role in lung cancer development: as an oncogene in early stages of lung cancer and as an inhibitor of lung cancer metastasis in advanced lung cancer stages [34]. miR-182-5p inhibits invasion and metastasis by regulating the EMT process through targeted down or up-regulation of ZEB2 (Zinc Finger E-Box Binding Homeobox 2), E-cadherin, and Vimentin proteins in NSCLC [39]. Overexpression of miR-182-3p may inhibit EMT and metastasis by inactivating the Met/ AKT/Snail pathway in NSCLC cells [40]. Therefore, studies should aim at elucidating on the expression and function of miR-182 in lung cancer metastasis.

In this study, miR-182-5p expression was associated with age, pathological stage, and tumor size and is, therefore, a potential independent prognostic factor for tumor recurrence and metastasis in NSCLC patients. In addition, overexpression of miR-182-5p promoted lung cancer cell invasion and metastasis in vitro. Besides, miR-182-5p upregulated the expression of Twist, MMP-2 (matrix metallopeptidase 2), MMP-9 (matrix metallopeptidase 9), N-cadherin, Vimentin, and Snail proteins, which promoted the EMT process in lung cancer. Downstream target genes of miR-182-5p, predicted using an online platform, revealed that EPAS1, a potential target gene for miR-182-5p, affects cell invasion and migration. This finding was confirmed using the luciferase report assay.

EPAS1 is a transcription factor that is responsible for inducing genes associated with cell survival under hypoxic conditions [41]. Studies have shown that EPAS1 promotes peritoneal carcinogenesis in NSCLC patients by enhancing MMT (mesothelialmesenchymal transition), therefore, it is a potential prognostic marker or therapeutic target for NSCLC [17]. Bioinformatics analysis of miR-182-5p revealed that EPAS1, PRKCE (protein kinase C epsilon), NR3C1 (nuclear receptor subfamily 3 group $C$ member 1), and RHOB are the primary genes in the protein-protein interaction (PPI) network of lung squamous cell carcinoma [18]. Extracellular ATP regulates EPAS1 and its target protein through the AKT-PGK1 pathway to inhibit breast cancer EMT [42]. In this study, EPAS1 was negatively correlated with miR-182-5p expression. Elevated miR-182-5p levels significantly down-regulated EPAS1 expression in vitro. Therefore, miR-182-5p promotes lung cancer invasion and metastasis by negatively regulating EPAS1. 


\section{Supplementary Material}

Supplementary figures and tables.

http://www.jcancer.org/v12p7120s1.pdf

\section{Acknowledgements}

\section{Funding}

This work was supported by National Natural Science Foundation of China (No.81704035), Shanghai Sailing Program (No.20YF1450800), Nation al Natural Science Foundation of China (No.81973810) and The YSN Science Program of the Shanghai University of Traditional Chinese Medicine (2019LK026).

\section{Author Contributions}

L. Jiao. and L. Xu. designed the research. W. Yang., Y. Yin. and Y. Wang. performed the experiments. W. Yang., Y. Yin., L. Bi., J. Yao. Y. Wang., and L. Jiao. analyzed the data. W. Yang. and Y. Yin. wrote the manuscript. L. Jiao. and L. Xu. edited the manuscript.

\section{Competing Interests}

The authors have declared that no competing interest exists.

\section{References}

1. Allemani C, Matsuda T, Di Carlo V, Harewood R, Matz M, Niksic M, et al. Global surveillance of trends in cancer survival 2000-14 (CONCORD-3): analysis of individual records for 37513025 patients diagnosed with one of 18 cancers from 322 population-based registries in 71 countries. Lancet (London, England). 2018; 391: 1023-75

2. Feng RM, Zong YN, Cao SM, Xu RH. Current cancer situation in China: good or bad news from the 2018 Global Cancer Statistics? Cancer communications (London, England). 2019; 39: 22

3. Wagner G, Stollenwerk HK, Klerings I, Pecherstorfer M, Gartlehner G, Singer J. Efficacy and safety of immune checkpoint inhibitors in patients with advanced non-small cell lung cancer (NSCLC): a systematic literature review. Oncoimmunology. 2020; 9: 1774314

4. Hammerschmidt S, Wirtz H. Lung cancer: current diagnosis and treatment. Dtsch Arztebl Int. 2009; 106: 809-18.

5. Bellavia D, Salamanna F, Raimondi L, De Luca A, Carina V, Costa V, et al. Deregulated miRNAs in osteoporosis: effects in bone metastasis. Cellular and molecular life sciences: CMLS. 2019; 76: 3723-44

6. Bartel DP. MicroRNAs: genomics, biogenesis, mechanism, and function. Cell. 2004; 116: 281-97.

7. Ma Y, Liang AJ, Fan YP, Huang YR, Zhao XM, Sun Y, et al. Dysregulation and functional roles of miR-183-96-182 cluster in cancer cell proliferation, invasion and metastasis. Oncotarget. 2016; 7: 42805-25.

8. Wang F, Wu D, Xu Z, Chen J, Zhang J, Li X, et al. miR-182-5p affects human bladder cancer cell proliferation, migration and invasion through regulating Cofilin 1. Cancer cell international. 2019; 19: 42

9. Rihani A, Van Goethem A, Ongenaert M, De Brouwer S, Volders PJ, Agarwal $\mathrm{S}$, et al. Genome wide expression profiling of p53 regulated miRNAs in neuroblastoma. Scientific reports. 2015; 5: 9027.

10. Cao MQ, You AB, Zhu XD, Zhang W, Zhang YY, Zhang SZ, et al. miR-182-5p promotes hepatocellular carcinoma progression by repressing FOXO3a. Journal of hematology \& oncology. 2018; 11: 12

11. Sachdeva M, Mito JK, Lee CL, Zhang M, Li Z, Dodd RD, et al. MicroRNA-182 drives metastasis of primary sarcomas by targeting multiple genes. The Journal of clinical investigation. 2014; 124: 4305-19.

12. Song $\mathrm{L}$, Liu L, Wu Z, Li Y, Ying Z, Lin C, et al. TGF-beta induces miR-182 to sustain NF-kappaB activation in glioma subsets. The Journal of clinical investigation. 2012; 122: 3563-78.

13. Ning FL, Wang F, Li ML, Yu ZS, Hao YZ, Chen SS. MicroRNA-182 modulates chemosensitivity of human non-small cell lung cancer to cisplatin by targeting PDCD4. Diagnostic pathology. 2014; 9: 143.

14. Zhang $\mathrm{Q}$, Lou $\mathrm{Y}$, Zhang J, Fu $\mathrm{Q}$, Wei $\mathrm{T}$, Sun $\mathrm{X}$, et al. Hypoxia-inducible factor-2a promotes tumor progression and has crosstalk with Wnt/ $\beta$-catenin signaling in pancreatic cancer. Molecular cancer. 2017; 16: 119
15. Gkotinakou IM, Kechagia E, Pazaitou-Panayiotou K, Mylonis I, Liakos P, Tsakalof A. Calcitriol Suppresses HIF-1 and HIF-2 Transcriptional Activity by Reducing HIF-1/2 $\alpha$ Protein Levels via a VDR-Independent Mechanism. Cells. 2020; 9: 2440.

16. Conrad PW, Freeman TL, Beitner-Johnson D, Millhorn DE. EPAS1 trans-activation during hypoxia requires $\mathrm{p} 42 / \mathrm{p} 44$ MAPK. The Journal of biological chemistry. 1999; 274: 33709-13.

17. Zhen Q, Zhang Y, Gao L, Wang R, Chu W, Zhao X, et al. EPAS1 promotes peritoneal carcinomatosis of non-small-cell lung cancer by enhancing mesothelial-mesenchymal transition. Strahlenther Onkol,2021:197(2):141-149.

18. Luo J, Shi K, Yin SY, Tang RX, Chen WJ, Huang LZ, et al. Clinical value of miR-182-5p in lung squamous cell carcinoma: a study combining data from TCGA, GEO, and RT-qPCR validation. World journal of surgical oncology. 2018; $16: 76$.

19. Kertesz M, Iovino N, Unnerstall U, Gaul U, Segal E. The role of site accessibility in microRNA target recognition. Nature genetics. 2007; 39: 1278-84

20. Vejnar CE, Zdobnov EM. MiRmap: comprehensive prediction of microRNA target repression strength. Nucleic acids research. 2012; 40: 11673-83.

21. Maragkakis M, Vergoulis T, Alexiou P, Reczko M, Plomaritou K, Gousis M, et al. DIANA-microT Web server upgrade supports Fly and Worm miRNA target prediction and bibliographic miRNA to disease association. Nucleic acids research. 2011; 39: W145-8.

22. Betel D, Koppal A, Agius P, Sander C, Leslie C. Comprehensive modeling of microRNA targets predicts functional non-conserved and non-canonical sites. Genome biology. 2010; 11: R90

23. Anders G, Mackowiak SD, Jens M, Maaskola J, Kuntzagk A, Rajewsky N, et al. doRiNA: a database of RNA interactions in post-transcriptional regulation. Nucleic acids research. 2012; 40: D180-6.

24. Grimson A, Farh KK, Johnston WK, Garrett-Engele P, Lim LP, Bartel DP. MicroRNA targeting specificity in mammals: determinants beyond seed pairing. Molecular cell. 2007; 27: 91-105.

25. Tang Z, Kang B, Li C, Chen T, Zhang Z. GEPIA2: an enhanced web server for large-scale expression profiling and interactive analysis. Nucleic acids research 2019: 47: W556-w60.

26. Li JH, Liu S, Zhou H, Qu LH, Yang JH. starBase v2.0: decoding miRNA-ceRNA, miRNA-ncRNA and protein-RNA interaction networks from large-scale CLIP-Seq data. Nucleic acids research. 2014; 42: D92-7.

27. Yang W, Kang Y, Zhao Q, Bi L, Jiao L, Gu Y, et al. Herbal formula Yangyinjiedu induces lung cancer cell apoptosis via activation of early growth response 1. Journal of cellular and molecular medicine. 2019; 23: 6193-202.

28. Zhu W, Zhou K, Zha Y, Chen D, He J, Ma H, et al. Diagnostic Value of Serum miR-182, miR-183, miR-210, and miR-126 Levels in Patients with Early-Stage Non-Small Cell Lung Cancer. PloS one. 2016; 11: e0153046.

29. Iqbal MA, Arora S, Prakasam G, Calin GA, Syed MA. MicroRNA in lung cancer: role, mechanisms, pathways and therapeutic relevance. Molecular aspects of medicine. 2019; 70: 3-20.

30. Zhang L, Liu T, Huang $Y$, Liu J. microRNA-182 inhibits the proliferation and invasion of human lung adenocarcinoma cells through its effect on human cortical actin-associated protein. International journal of molecular medicine. 2011; 28: 381-8.

31. Yang $\mathrm{O}$, Tang $\mathrm{Y}$, Tang $\mathrm{C}$, Cong $\mathrm{H}$, Wang $\mathrm{X}$, Shen $\mathrm{X}$, et al. Diminished LINC00173 expression induced miR-182-5p accumulation promotes cell proliferation, migration and apoptosis inhibition via AGER/NF- $\mathrm{kB}$ pathway in non-small-cell lung cancer. American journal of translational research. 2019; 11: 4248-62.

32. Li Y, Zhang H, Gong H, Yuan Y, Li Y, Wang C, et al. miR-182 suppresses invadopodia formation and metastasis in non-small cell lung cancer by targeting cortactin gene. Journal of experimental \& clinical cancer research : CR. 2018; 37: 141.

33. Li P, Sheng C, Huang L, Zhang H, Huang L, Cheng Z, et al. MiR-183/-96/-182 cluster is up-regulated in most breast cancers and increases cell proliferation and migration. Breast cancer research : BCR. 2014; 16: 473

34. Yang WB, Chen PH, Hsu Ts, Fu TF, Su WC, Liaw H, et al. Sp1-mediated microRNA-182 expression regulates lung cancer progression. Oncotarget. 2014; 5: 740-53.

35. Dettmer M, Vogetseder A, Durso MB, Moch H, Komminoth P, Perren A, et al. MicroRNA expression array identifies novel diagnostic markers for conventional and oncocytic follicular thyroid carcinomas. The Journal of clinical endocrinology and metabolism. 2013; 98: E1-7.

36. Weeraratne SD, Amani V, Teider N, Pierre-Francois J, Winter D, Kye MJ, et al. Pleiotropic effects of miR-183 96 182 converge to regulate cell survival, proliferation and migration in medulloblastoma. Acta neuropathologica. 2012; 123: 539-52.

37. Kouri FM, Hurley LA, Daniel WL, Day ES, Hua Y, Hao L, et al. miR-182 integrates apoptosis, growth, and differentiation programs in glioblastoma. Genes \& development. 2015; 29: 732-45.

38. Abd-El-Fattah AA, Sadik NA, Shaker OG, Aboulftouh ML. Differential microRNAs expression in serum of patients with lung cancer, pulmonary tuberculosis, and pneumonia. Cell biochemistry and biophysics. 2013; 67: 875-84.

39. Sun Y, Fang R, Li C, Li L, Li F, Ye X, et al. Hsa-mir-182 suppresses lung tumorigenesis through down regulation of RGS17 expression in vitro. Biochemical and biophysical research communications. 2010; 396: 501-7. 
40. Li Y, Zhang H, Li Y, Zhao C, Fan Y, Liu J, et al. MiR-182 inhibits the epithelial to mesenchymal transition and metastasis of lung cancer cells by targeting the Met gene. 2018; 57: 125-36.

41. Cam H, Easton JB, High A, Houghton PJ. mTORC1 signaling under hypoxic conditions is controlled by ATM-dependent phosphorylation of HIF-1a. Molecular cell. 2010; 40: 509-20.

42. Yang $\mathrm{H}$, Geng $\mathrm{YH}$, Wang $\mathrm{P}$, Zhou YT, Yang H, Huo YF, et al. Extracellular ATP promotes breast cancer invasion and epithelial-mesenchymal transition via hypoxia-inducible factor 2alpha signaling. 2019; 110: 2456-70 